\title{
MEASUREMENT AND SIMULATION OF ANTHROPOGENIC AND BIOGENIC VOC EMISSIONS FROM DIFFERENT TYPES OF SOURCES
}

\author{
CORINNA BARRACO, MARCO RAVINA, DEBORAH PANEPINTO \& MARIACHIARA ZANETTI \\ DIATI Department, Politecnico di Torino, Italy
}

\begin{abstract}
The aim of this work is to find a methodology in order to distinguish the anthropogenic and biogenic emissions of the following compounds in the atmosphere: Volatile Organic Compounds emitted by an anthropic source (VOC) and Biological Volatile Organic Compounds (BVOC). The implemented methodology consists of two phases: the first one foresees measurements that were taken to simultaneously record the natural and anthropic contribution, the second one foresees the implementation of a Matlab program called bioVOC in order to simulate the emission of only the natural contribution. The study areas chosen are Bardonecchia (a long way from the Turin urban zone) and Settimo Torinese (close to the Turin urban zone), in the Piedmont region of Italy. BioVOC model needs the Leaf Area Index (LAI), the environmental temperature $(\mathrm{K})$, the solar radiation $(\mu \mathrm{mol} / \mathrm{J})$ and the land use with Corine classification as input, and returns the hourly flow emitted by the vegetation $\left(\mu \mathrm{g} / \mathrm{m}^{3} \mathrm{~h}\right)$. The validation of the model took place by comparison with SurfPRO, an existing model that achieves the same results as bioVOC but is based on different concepts. Through the measurements made, it was found that there is a much higher concentration of the natural and anthropic contribution in Settimo Torinese than in Bardonecchia (305 ppb versus $135 \mathrm{ppb}$ ). On the other hand the BVOC flow simulated with the implemented Matlab model provides completely different results. The biogenic emission simulated in Settimo Torinese is negligible compared to that simulated in Bardonecchia $\left(0.02 \mu \mathrm{g} / \mathrm{m}^{2} \mathrm{~h}\right.$ versus $\left.2.7 \mu \mathrm{g} / \mathrm{m}^{2} \mathrm{~h}\right)$, so the concentration measured with the instrument in Settimo Torinese is mostly attributable to the anthropogenic contribution. In conclusion, the applied method is able to distinguish the natural contribution from the anthropic one.
\end{abstract}

Keywords: VOCs, modeling, air quality, North Italy.

\section{INTRODUCTION}

The quantitative estimate of global BVOC emissions into the atmosphere began with the fundamental work of Went [1] who extrapolated the monoterpenes measurements from a single plant species [2].

Subsequently, Rasmussen et al. [3] observed that each plant species has a different ability to emit BVOC and therefore divided the biosphere into groups of plants based on the type of emission. Zimmerman et al. [4] extended this approach to the use of more comprehensive land cover data and preferred to divide western forests into four types, depending on the terpene emitted in larger quantities. The four categories are: high isoprene deciduous forest, low isoprene deciduous forest, zero isoprene deciduous forest and coniferous forest.

The study on VOC emissions subsequently focused on the interaction with urban activities. The quality of the absorbed urban pollutants or the emitted BVOCs vary according to the species [5] and the climatic conditions, such as the temperature and the concentration of pollutants in the atmosphere [6].

The quantity of urban pollutants absorbed or BVOC emitted also depends on plant species and environmental conditions. In the case of particulate matter, for example, the species of conifers, or in general species with a complex leaf system, have a greater capacity to absorb pollutants, as demonstrated in various studies [7], [8]. 
This study aims to find a method capable of distinguishing VOCs of anthropic origin from biogenic ones and, therefore, a comparison is made between the measured and simulated values in Bardonecchia and Settimo Torinese (Piedmont, Italy), in order to obtain the composition of emissions.

\section{METHODOLOGY}

The method consists of several stages. First of all, it is necessary to measure the concentration of BVOC and VOC in natural and urban environments; in addition, it will be necessary to implement a model capable of simulating biogenic emissions and applying it to the study area. Once the two previous phases have been completed, it will be possible to reach a conclusion by comparing the results obtained.

\subsection{Measurements}

The purpose of the measurements is to know the total concentration of both anthropogenic and natural VOCs, in a natural environment and also in an urban one.

Bardonecchia was chosen to measure the natural contribution because it is sufficiently distant from the anthropic construct and it is close to a meteorological station (Pian del Sol, altitude $1300 \mathrm{~m}$, coordinates 319383, 4992738). For the construction of the model, it is essential to know the weather conditions because temperature, humidity and atmospheric pressure are factors that influence the emission of terpenes.

The location where the anthropogenic contribution was taken is Settimo Torinese because it is close to an industrial area and to the metropolitan city of Turin, a strongly anthropized area. Once turned on, the TIGER ION instrument used for measurement has stored a concentration value in ppb every 2 seconds, for the entire measurement time.

\subsection{Description of the implemented model}

First of all, below is a description of the references followed for the implementation on Matlab of the model called bioVOC. Megan is the model whose equations were used for the implementation of bioVOC and finally SURFPro was used for the validation.

\subsubsection{Megan emission model}

The MEGAN v2.1 model [9] assigns emission factors and parameters to 19 BVOC classes for each of the 15 types of land cover (PFT) used for the community land model (CLM4), according to the equation

$$
F_{i}=\gamma_{i} * \sum \varepsilon_{i j} x_{j}
$$

where $\varepsilon_{i j}$ is the emission factor at standard conditions for the type of vegetation $\mathrm{j}$, with area coverage with fractional grid $\mathrm{x}_{\mathrm{j}}$. The emission activity factor (i) represents the processes that control the emission responses to environmental and phenological conditions. The two main components of the model are the algorithms that describe the emission according to the environmental conditions and the main processes. These processes include an electron transport-based light response for light-dependent emissions, a temperature response based on enzymatic activity and $\mathrm{CO}_{2}$ response [10].

The activity factor $\left(\gamma_{i}\right)$ for each class of compounds takes into account the emission response to light $\left(\gamma_{P}\right)$, temperature $\left(\gamma_{T}\right)$, leaf age $\left(\gamma_{A}\right)$, soil moisture $\left(\gamma_{S M}\right)$, Leaf Area Index (LAI) and $\mathrm{CO}_{2}$ inhibition $\left(\gamma_{C}\right)$, and it is estimated as

$$
\gamma_{i}=C_{C E} L A I \gamma_{P, i} \gamma_{T, i} \gamma_{A, i} \gamma_{S M, i} \gamma_{C, i}
$$


where $C_{C E}$ is the coefficient relative to the canopy environment, which is 0.30 for the crown model CLM4.

\subsubsection{SURFPro emission model}

SURFace-atmosphere interface PROcessor, Version 3 is a tool developed to estimate horizontal and vertical spreads, ground deposition rates according to the type of soil cover (Corine classification) and the characteristics of the chemical species.

The rate of emission of a species from an i-th terrestrial ecosystem into the atmosphere, at a specific location and time, is calculated using the following equation [11]

$$
E_{s, i}=\varepsilon_{s, i} * \gamma_{s} * D_{i} * f_{i},
$$

where $\varepsilon_{s, i}$ (in $\mu g / g * h$ ) is the emission speed of the compound in the canopy $i$ under standard conditions, $\gamma_{\mathrm{s}}$ (normalized ratio) is an environmental correction factor due to temperature and photosynthetically active radiation (PAR) (dimensionless), $D_{i}$ and $f_{i}$ are the density of the leaf biomass $\left(\mathrm{g} / \mathrm{m}^{2}\right)$ and the fraction covered by the terrestrial ecosystem respectively.

\subsection{The bioVOC model implementation}

The realization of bioVOC model able to predict the flow of terpenes emitted by the natural contribution in a specific area, follows the Megan2.1 algorithm, reported in the previous paragraph. Fig. 1 shows the functional diagram of the model bioVOC.

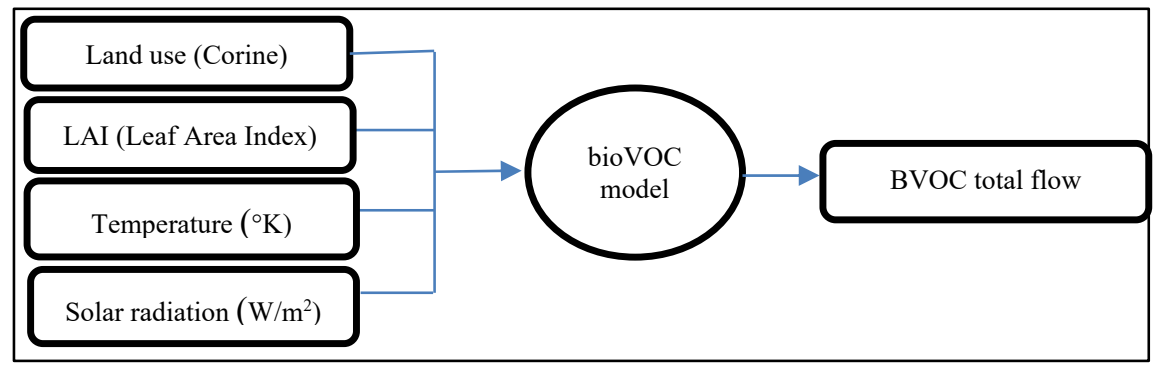

Figure 1: The bioVOC functional scheme.

Among the input data, temperature and radiation are available from the nearby meteorological station (for Bardonecchia) or on the website www.arpa.piemonte.it (for Settimo Torinese), while the LAI is found in literature. The land use is necessary because each type of vegetation has a different emission factor relative to each of the different BVOCs it can emit, so it is necessary to divide the area by means of a grid into cells, in which only the prevailing type of vegetation will be considered. The classification chosen for land use is Corine because it is also used in SurfPRO (useful for validation) and subsequently it is converted into the CLM PFT classification with which the relative emission factors are associated.

\subsection{Model validation}

Once the Matlab model was completed, it was decided to validate it by comparing the results obtained by SurfPRO. 
From the comparison there will not be exactly the same values because the models are based on different considerations and equations, as shown in the previous paragraph.

This comparison is useful to check that the orders of magnitude are similar because this is sufficient to make the results obtained by bioVOC plausible.

As can be seen from Fig. 2, the total isoprene and VOC values calculated by the two models are very similar to each other. The validation confirms, therefore, the consistency of the results of the developed model.

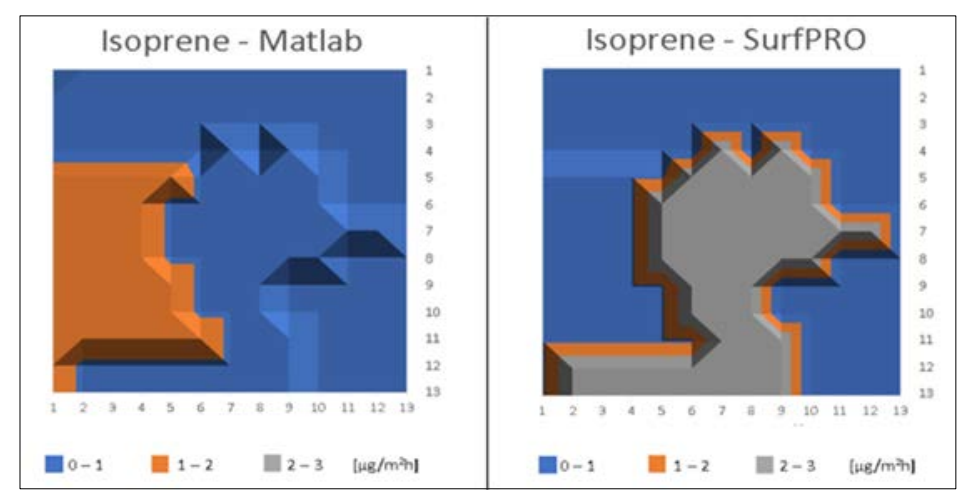

Figure 2: Validation of the bioVOC model by comparing results with SurfPro.

\section{RESULTS}

The results obtained with the TIGER measuring instrument and calculated with the bioVOC model developed are discussed, for both sites. Specifically, in Settimo Torinese only the area covered by vegetation is considered to simulate the emission, since it is the only one that emits BVOC that can be calculated with bioVOC model.

In this way it will be possible to obtain useful information on the dynamics that involve the emission of BVOCs from the natural contribution.

\subsection{Measurements results}

The average of VOCs measured in Bardonecchia is $135 \mathrm{ppb}$ (Fig. 3). As for Settimo Torinese, very different results have been obtained (Fig. 4). First of all, in Settimo Torinese the average is $305 \mathrm{ppb}$ and a very high concentration peak was measured at the beginning of measures (Fig. 5). This peak is impossible to find even in the natural environment, in fact the contribution of plant species is always about constant (except for seasonal variations).

A direct comparison between Bardonecchia and Settimo Torinese is shown in Fig. 6, where it is possible to evaluate that VOC concentration measured in Settimo Torinese is almost double that measured in Bardonecchia, but the question is: what percentage of VOC detected by the instrument is of anthropogenic origin and which biogenic? These questions can be answered with the support of the biogenic emission simulation model.

\subsection{Model results}

The following figures show the flow predicted by bioVOC model regarding the natural contribution on the measurement days (in $\mu \mathrm{g} / \mathrm{m}^{2} \mathrm{~h}$ ), for Bardonecchia (Fig. 7) and Settimo Torinese (Fig. 8). 




Figure 3: Measurements results for Bardonecchia.

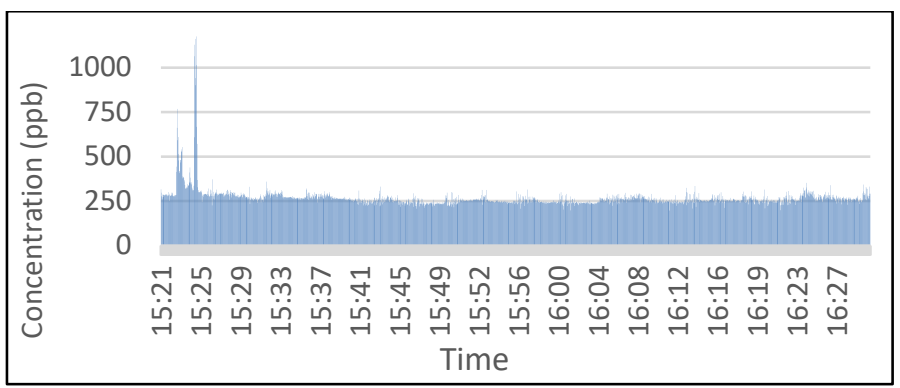

Figure 4: Measurements results for Settimo Torinese.



Figure 5: Concentration peak in Settimo Torinese.

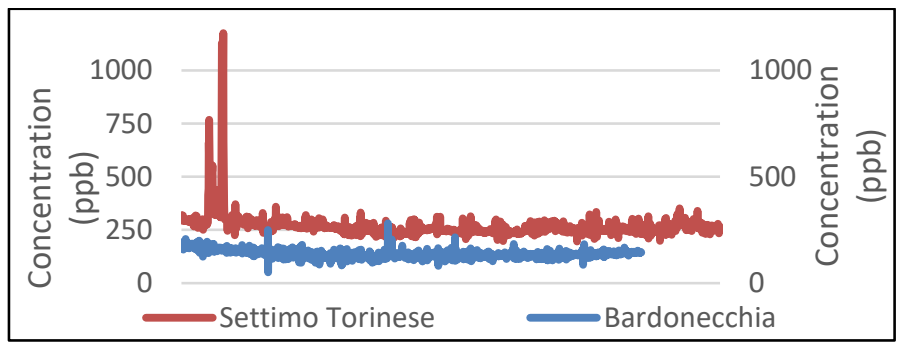

Figure 6: Comparison between Bardonecchia and Settimo Torinese results. 




Figure 7: Emission flow estimated by bioVOC in Bardonecchia.

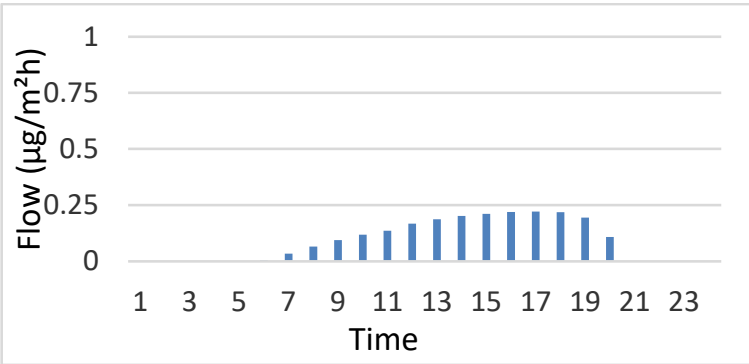

Figure 8: Emission flow estimated by bioVOC in Settimo Torinese.

In Settimo Torinese, the presence of plant species is very small, in fact here the contribution of VOC derives mainly from the industrial constructs and from the processes taking place at different times of the day.

The two curves have very different values but similar shapes, as they have the maximum flow value emitted around $4 \mathrm{pm}$ (time when the heat accumulated by the day peaks in June in northern Italy) and, instead, at night they do not report emissions. This is given by the fact that the temperature is the parameter that most conditions the emission of VOC from the vegetation.

\subsubsection{Bardonecchia}

To appreciate even more the role of temperature in the terpene emission, refer to Fig. 9, in which it is possible to observe how the emission changes in correspondence of 5 days in which the temperature has been increasing day by day. In this figure, in fact, it is possible to note how an increment of $+3^{\circ} \mathrm{C}$ of minimum temperature value and $+5^{\circ} \mathrm{C}$ of maximum, from 31 May to 6 Jun. 2019, can even triple the values of the total flow emitted. But temperature is not the only factor that regulates the emission of VOCs, the type of vegetation considered plays a fundamental role in addition to the temperature, because each plant species is able to emit different quantities and types of BVOC.

The types of plant species present in Bardonecchia are coniferous forests, mixed forests and shrubs and heathlands. For each of these vegetation types, scientific coefficients are assigned which indicate the quantity and type of BVOC emitted. In this study, three categories of terpenes are taken into consideration: isoprene, the sum of all monoterpenes and the total VOC flow, which is the sum of the first two contributions. 


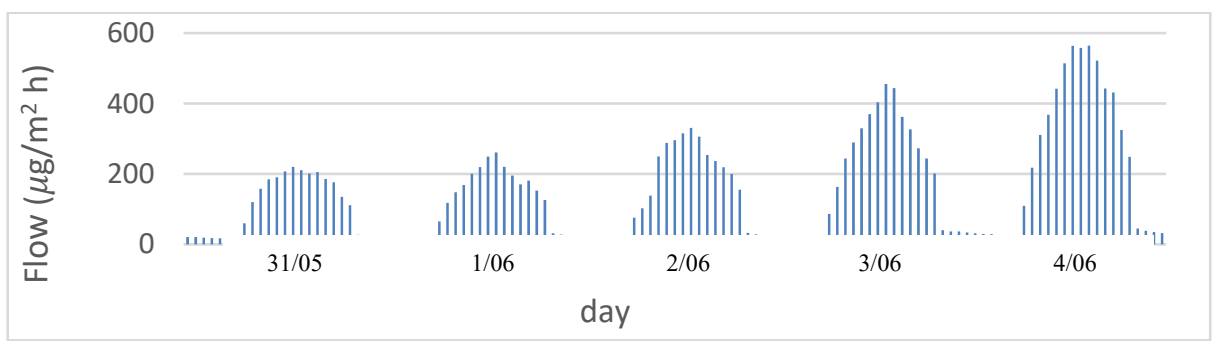

Figure 9: Total flow Bardonecchia and dependence on temperature.

For each of the three plant species considered, Fig. 10 shows the flow of isoprene (the most important and common BVOC) in Bardonecchia, and it is interesting to emphasize that the three curves, each relating to a type of vegetation, all have the same trend, but different values. This means that the quality of the emission is the same and therefore does not depend on the plant species, but on temperature, radiation and the other environmental parameters, which are the same for all types of vegetation. As for the quantity of the emission, instead, there are very different values from one plant species to another. In the case of isoprene, the mixed forests are the main ones responsible for its introduction into the atmosphere, on the contrary coniferous forests contribute less.

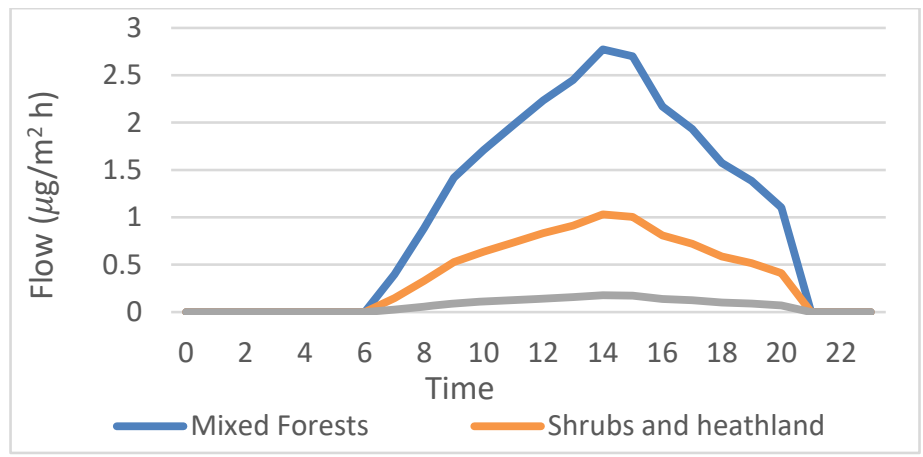

Figure 10: Isoprene flow emitted in Bardonecchia.

In accord to eqn (1), it is possible to pass from the parameter $\gamma_{i}$ to the emitted flow $\left(F_{i}\right)$ through the emission factor $\left(\varepsilon_{i j}\right)$ that is related to the plant species. For eqn (2), the $\gamma_{i}$ value depends on environmental conditions. This means that $\gamma_{i}$ is the quantity of emission available in specific environmental conditions, and finally the specific emission factor of the plant species contributes to decreasing or increasing its value depending on whether the given plant species emits much or little of the specific terpene considered.

In Figs 11 and 12, the value of $\gamma_{\text {isoprene }}$ is lower than that of the isoprene flow and this information confirms the fact that both types of vegetation emit a lot of isoprene. Instead, Fig. 13 shows that the initial value of $\gamma_{\text {isoprene }}$ is higher than the emitted isoprene flow, this means that, with the same environmental conditions, coniferous forests never emit as much isoprene as the other types analysed. 




Figure 11: Mixed Forests emitted flow in Bardonecchia.

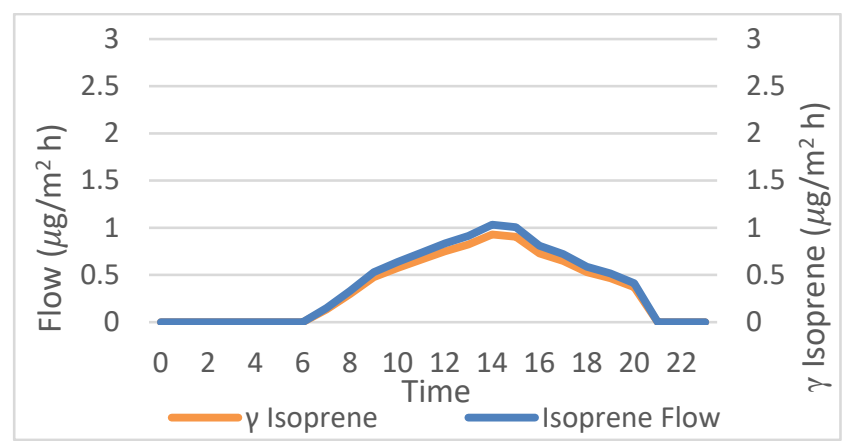

Figure 12: Shrubs and headlands emitted flow in Bardonecchia.

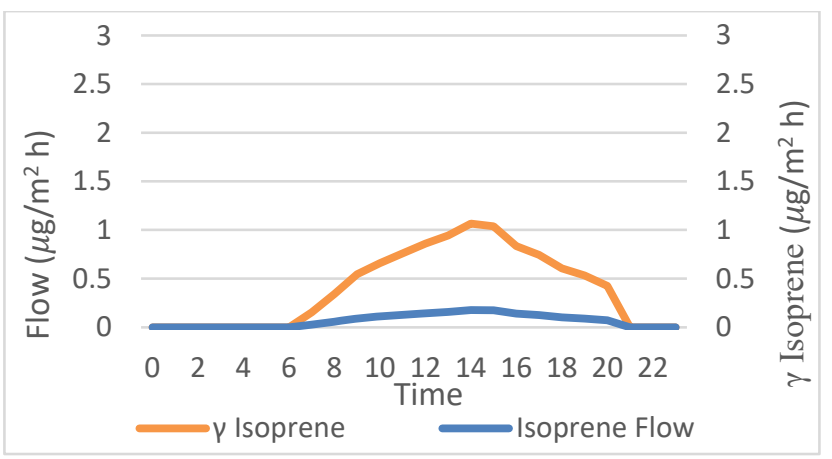

Figure 13: Coniferous Forests emitted flow in Bardonecchia.

\subsubsection{Settimo Torinese}

The only plant species present in Settimo Torinese are crops, which are not evenly distributed over the entire area considered. The three curves of Fig. 14 show the quality of the emission from Settimo Torinese crops, highlighting in what proportions isoprene, monoterpenes and total VOCs are emitted. As can be seen in the figure, crops emit mainly monoterpenes and instead the contribution of isoprene is negligible. Not only that, a substantial difference, 


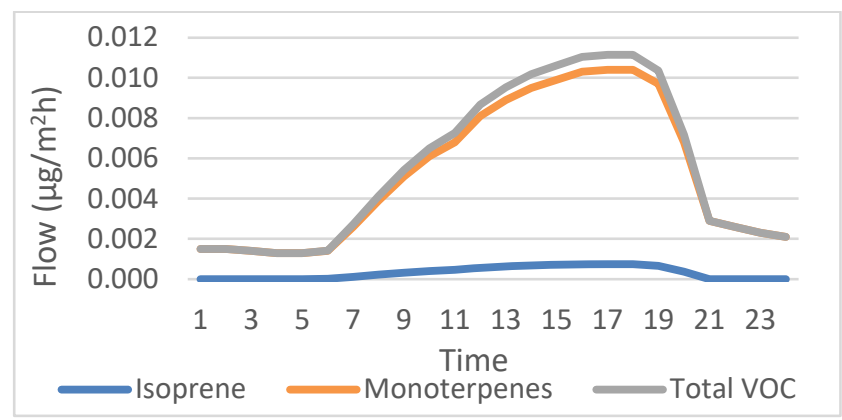

Figure 14: Isoprene, monoterpenes and total VOC emission in Settimo Torinese by crops.

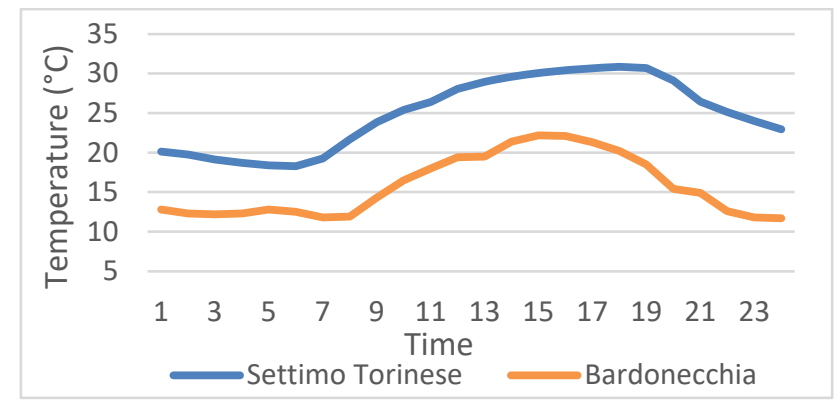

Figure 15: Temperature in Settimo Torinese and Bardonecchia (19/06/2019).

compared to the figures obtained for Bardonecchia and discussed in the previous paragraph, lies in the fact that for Settimo Torinese the values are 3 orders of magnitude lower.

Temperature is the factor that most influences the emission of BVOC by vegetation, therefore it is advisable to compare the temperature recorded in Settimo Torinese and Bardonecchia on the day of measurement (Fig. 15).

The temperature in Settimo Torinese is several degrees higher than that recorded in Bardonecchia, in addition to the different altitude of the two sites, probably due to the fact that the urban buildings present in the Settimo Torinese site accumulate more heat than only vegetation present in Bardonecchia. For this reason, the issue of BVOC in Settimo Torinese is more favoured than in Bardonecchia. However, this does not mean that the BVOC contribution issued is greater in Settimo Torinese; in fact, although here the environmental conditions that favour the emission of terpenes are better than Bardonecchia, the plant species present have a too low emission factor and, moreover, the plant species do not cover the entire area uniformly, as in the case of Bardonecchia. Consequently, in Settimo Torinese there is a much lower contribution of biogenic VOC emissions than in Bardonecchia.

\subsubsection{Comparison between Bardonecchia and Settimo Torinese}

The contributions of each of the two sites are compared in the same figure, divided between isoprene emission (Fig. 16) and monoterpenes (Fig. 17). 


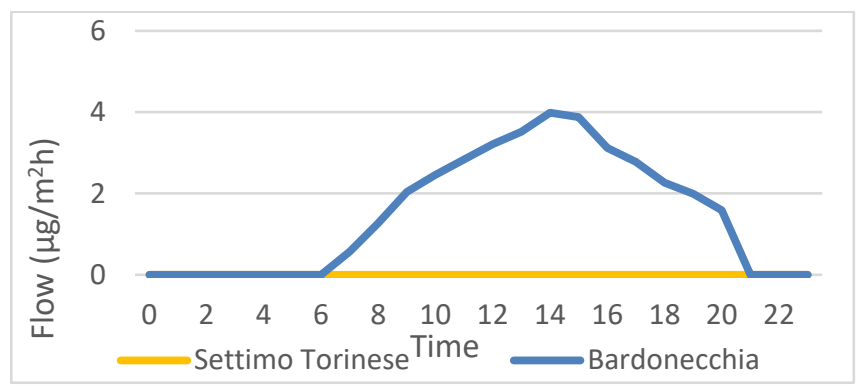

Figure 16: Isoprene emission in Bardonecchia and Settimo Torinese.

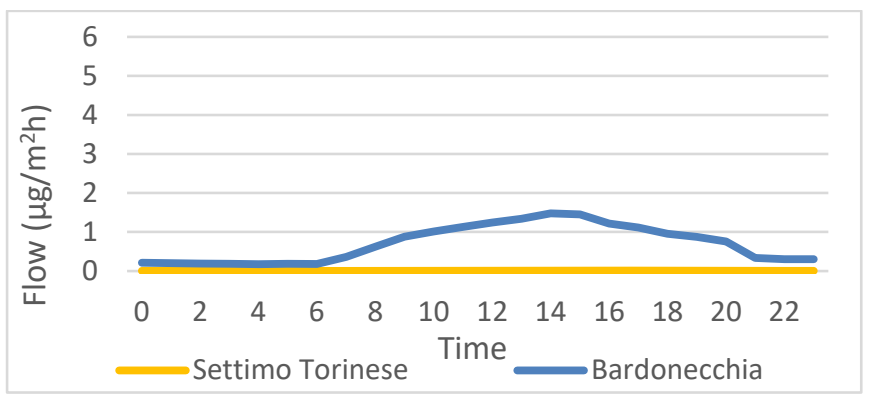

Figure 17: Monoterpenes emission in Bardonecchia and Settimo Torinese.

From Fig. 16, it is clear that the contribution of isoprene by Settimo Torinese is null compared to that which one has in Bardonecchia. This is mainly due to the fact that the cultivations present in Settimo Torinese have a low emission factor compared to the plant species present in Bardonecchia.

Fig. 17 compares the emissions of monoterpenes between the two sites, one should therefore appreciate a less marked difference between the contributions of Settimo Torinese and Bardonecchia, since the plant species present in Settimo Torinese mostly emits monoterpenes. In reality, even in this case the contribution of monoterpenes in Settimo Torinese is negligible compared to Bardonecchia.

To ascertain what the main cause is, it is advisable to compare the values of the $\gamma_{\text {isoprene }}$ for plant species present at Settimo Torinese and Bardonecchia sites. The value of $\gamma_{\text {isoprene }}$ encompasses all the specific environmental characteristics of the site. A high value of $\gamma_{\text {isoprene }}$ means that the environmental conditions at that site are optimal for the emission of BVOCs by vegetation.

Fig. 18 shows that in Settimo Torinese the value of $\gamma_{\text {isoprene }}$ is much higher than in Bardonecchia, which means that the environmental conditions are better in Settimo Torinese than in Bardonecchia and consequently the plant species present are more stimulated to release $\mathrm{BVOC}$ into the atmosphere. 


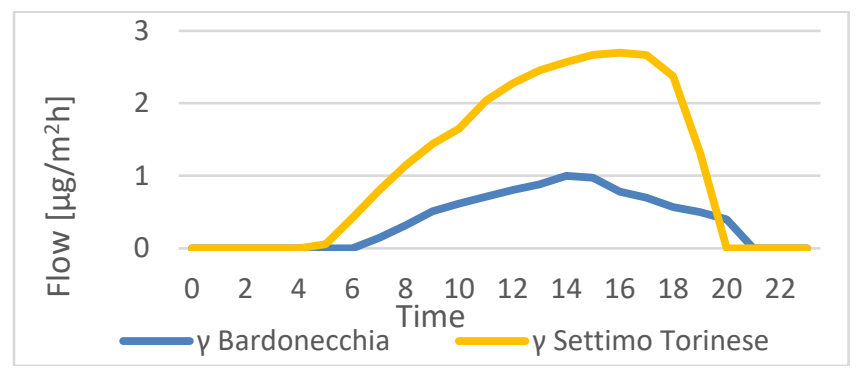

Figure 18: Comparison between $\gamma_{\text {isoprene }}$ in Settimo Torinese and Bardonecchia.

\section{CONCLUSIONS}

The study aims to distinguish the anthropic contribution from the natural one, in the emission of VOCs in the atmosphere. To achieve this goal, it was necessary to make measurements of the total contribution, both anthropogenic and natural, and to develop a Matlab model capable of calculating the natural contribution of the study areas.

The areas in which the measurements were made are the same for which the emission values were calculated with the Matlab model bioVOC. These areas are Bardonecchia, characterized by vegetation, and Settimo Torinese, mainly characterized by urban construction.

Once the model able to predict the emission of biogenic VOCs by vegetation was validated, it was possible to calculate the natural emission values of the two study zones in order to compare them with the relative concentration values measured by the instrument.

From the comparison between the values recorded by the TIGER measuring instrument for the two sites, it results that in Settimo Torinese there is an almost double background concentration compared to Bardonecchia. It is expected to obtain similar results even with the Matlab model developed to calculate the natural contribution.

In reality, the results calculated with bioVOC are very different from those obtained with the TIGER measuring instrument. In fact, it emerges that the emission of BVOC by the plant species present in Settimo Torinese is far lower than the emission calculated on the Bardonecchia site, so as to be almost negligible.

Since the plant species present on Settimo Torinese emit an almost zero amount of BVOC compared to that calculated with the Matlab model for Bardonecchia, but at the same time the measurements reveal a completely opposite result, it can be concluded by saying that in Settimo Torinese the VOC contribution recorded by the measuring instrument is almost completely anthropogenic. In this area, if it is necessary to monitor the possible sources, or if it is necessary to investigate the composition of the VOCs to understand if dangerous substances are present in not negligible quantities, it is advisable to start from the assumption that the natural contribution in this area is almost null.

To further improve the analysis, it would be necessary to develop a dispersion model able to consider the microclimatic conditions of the study area and which knows how to calculate the relative concentration value on the ground.

\section{REFERENCES}

[1] Went, F.W., Blue hazes in the atmosphere. Nature, 187(4738), 1960. 
[2] Guenther, A., Biological and chemical diversity of biogenic volatile organic emissions into the atmosphere. ISRN Atmospheric Sciences, 2013, 786290, 27 pp., 2013. http://dx.doi.org/10.1155/2013/786290.

[3] Rasmussen, R., Baker, B., Jardine, K. \& Nemitz, E., The bi-directional exchange of oxygenated VOCs between a loblolly pine (Pinus Taeda) plantation and the atmosphere. Atmospheric Chemistry and Physics, 5, pp. 3015-3031, 2005.

[4] Zimmerman P.R., Greenberg, J.P. \& Westberg, C.E., Measurements of atmospheric hydrocarbons and biogenic emission fluxes in the Amazon Boundary Layer. Journal of Geophysical Research, 93(2), pp. 1407-1416, 1988.

[5] Steinbrecher, R. et al., Intra- and inter-annual variability of VOC emissions from natural and semi natural vegetation in Europe and neighbouring countries. Atmospheric Environment, 43, pp. 1380-1391, 2009.

[6] Calfapietra, C., Fares, S., Manes, F., Morani, A., Sgrigna, G. \& Loreto, F., Role of biogenic volatile organic compounds (BVOC) emitted by urban trees on ozone concentration in cities: A review. Environmental Pollution, 183, pp. 71-80, 2013. http://dx.doi.org/10.1016/j.envpol.2013.03.012.

[7] Beckett, K.P., Freer, P.H. \& Taylor, G., Particulate pollution capture by urban trees: effect of species and windspeed. Global Change Biology, 6, 995e1003, 2000.

[8] Freer-Smith, P.H., Beckett, K.P. \& Taylor, G., Deposition velocities to Sorbus aria, Acer campestre, Populus deltoides x trichocarpa "Beaupré", Pinus nigra and Cupressocyparis leylandii for coarse, fine and ultra-fine particles in the urban environment. Environmental Pollution, 133, 157e167, 2005.

[9] MEGAN version 2.10 User's Guide. www.lar.wsu.edu/megan/docs/MEGAN2.1_User_GuideWSU.pdf.

[10] Guenther, A.B., Monson, R.K. \& Fall, R., Isoprene and monoterpene emission rate variability: Observations with eucalyptus and emission rate algorithm development. Journal of Geophysical Research: Atmospheres, 96, pp. 10799-10808, 1991.

[11] Guenther, A.B., Zimmerman, P.R., Harley, P.C., Monson, R.K. \& Fall, R., Isoprene and monoterpene emission rate variability: Model evaluations and sensitivity analyses. Journal of Geophysical Research: Atmospheres, 98, pp. 12609-12617, 1993. 